\title{
DISTRIBUTIOONS AND THE LIE ALGEBRAS THEIR BASES CAN GENERATE
}

\author{
HENRY HERMES
}

(Communicated by Kenneth R. Meyer)

\begin{abstract}
The problem is to determine when a smooth, $k$-dimensional distribution $D^{k}$ defined on an $n$-manifold $M^{n}$, locally admits a vector field basis which generates a nilpotent, solvable or even finite-dimensional Lie algebra. We show that for every $2 \leq k \leq n-1$ there exists a (nonregular at $p \in M^{n}$ ) distribution $D^{k}$ on $M^{n}$ which does not locally (near $p$ ) admit a vector field basis generating a solvable Lie algebra. From classical results on the equivalence problem, it is shown that for $1 \leq k \leq 4$ and $D^{k}$ regular at $p \in M^{4}$, $D^{k}$ admits a local vector field basis generating a nilpotent Lie algebra.
\end{abstract}

\section{INTRODUCTION}

Let $x \rightarrow D^{k}(x)$ be a real analytic, $k$-distribution defined on a $n b d, U(p)$, of a point $p$ on the real analytic, $n$-dimensional manifold $M^{n}$. For $\left\{X^{1}, \ldots\right.$, $\left.X^{k}\right\}$ a local vector field basis of $D^{k}$ let $L=L\left(X^{1}, \ldots, X^{k}\right)$ denote the Lie algebra these vector fields generate. The problem considered is to determine when a distribution $D^{k}$ admits a basis generating a nilpotent, solvable, or finitedimensional Lie algebra (hereafter termed a nilpotent, solvable, or finite basis for $D^{k}$ ). The main result, Theorem 3, leads to the fact that for every $2 \leq k \leq$ $n-1$ there exists a $k$-distribution $D^{k}$ on $M^{n}$ which does not admit (locally) a solvable basis.

Let $D^{k}, E^{k}$ be $k$-distributions defined, respectively, on $n$-manifolds $M$, $N$. Classically, $D^{k}(p)$ is equivalent to $E^{k}(q)$ if there is a local diffeomorphism $\phi: M \rightarrow N$, defined on a $n b d \quad U(p) \subset M$, such that $\phi(p)=q$ and $\phi_{*}(x) D^{k}(x)=E^{k}(\phi(x))$ for $x \in U(p)$. We shall say $D^{k}(p)$ is self-equivalent to $D^{k}(q)$ if there exists a diffeomorphism $\phi$, defined on a $n b d . U(p)$, with $\phi(p)=q$ and $\phi_{*}(x) D^{k}(x)=D^{k}(\phi(x))$ for $x \in U(p)$. In [1], it was shown that if $D^{k}$ admits a local nilpotent basis near $p$, then every $n b d$ of $p$ contains a point $q \neq p$ such that $D^{k}(p)$ and $D^{k}(q)$ are self-equivalent. In this

Received by the editors March 6, 1988 and, in revised form, December 2, 1988.

1980 Mathematics Subject Classification (1985 Revision). Primary 58A30, 53A55.

This research was supported by NSF grant DMS-8500941 and by a visiting membership at the Mathematical Sciences Research Institute. 
case certain diffeomorphism invariants of distributions led to obstructions to the existence of local nilpotent bases.

In the solvable case, self-equivalence no longer need hold. We next describe, briefly, the procedure used to produce obstructions to the existence of a solvable basis.

For a Lie algebra $L$ of vector fields on an $n$-manifold $M^{n}$ with tangent space at $x$ denoted $T M_{x}^{n}$, let $L(p)=\left\{X(p) \in T M_{p}^{n}: X \in L\right\}$ and $L_{p}$ denote the isotropy algebra at $p$, i.e. $L_{p}=\{X \in L: X(p)=0\}$. Then $L$ is locally transitive at $p$ if $\operatorname{dim} L(p)=n$. For $D^{k}$ a local $k$-distribution on $M^{n}$ its $j$ th derived distribution is denoted $D^{k, j}$ and defined as follows. Choose any local basis $\left\{X^{1}, \ldots, X^{k}\right\}$ for $D^{k}$. Then $D^{k, j}(x)$ is the span of all products of $i$-tuples of elements from $\left\{X^{1}, \ldots, X^{k}\right\}$, evaluated at $x$, with $i \leq j+1$. This is a basis free notion. Note that $D^{k, 0}=D^{k}$ while for $j \geq 1, D^{k, j}$ may be a singular distribution, i.e. $\operatorname{dim} D^{k, j}(x)$ need not be constant in a $n b d$ of $p$. The integers $\operatorname{dim} D^{k, j}(x)$ are, however, diffeomorphism invariants, specifically if $D^{k}(p)$ is equivalent to $E^{k}(q)$, then $\operatorname{dim} D^{k, j}(p)=\operatorname{dim} E^{k, j}(q)$ for all $j=1,2, \ldots$.

In the case of a locally transitive, solvable, algebra $L$, we first show (Theorem 2) that every $n b d$ of $p$ contains a point $q \neq p$ such that the isotropy subalgebras $L_{p}, L_{q}$ are isomorphic and this isomorphism extends to an automorphsim $\bar{l}$ of $L$. By a theorem of Nagano-Sussmann, $[2,3]$, here stated as Theorem 1 , this implies that $D^{k}(p)$ and $\left(\bar{l} D^{k}\right)(q)=\operatorname{span}\left\{\left(\bar{l} X^{1}\right)(q), \ldots,\left(\bar{l} X^{k}\right)(q)\right\}$ are equivalent. Theorem 3 then shows that for $q$ sufficiently near $p$ the dimensions of the $j$ th derived distributions of $D^{k}$ and $\bar{l} D^{k}$ are the same at $q$ for all $j=1,2, \ldots$. Thus if $D^{k}$ has a local solvable basis every $n b d$ of $p$ contains a point $q \neq p$ such that $\operatorname{dim} D^{k, j}(p)=\operatorname{dim} D^{k, j}(q), i=1,2, \ldots$ Example 1 constructs a $D^{k}$ such that $\operatorname{dim} D^{k, 2}(x)>\operatorname{dim} D^{k, 2}(p)$ for all $x \neq p$ in some $n b d$ of $p$. Such a $D^{k}$ cannot admit a solvable basis. (One should note, however, that this behavior does not provide an obstruction to the existence of a basis generating a finite-dimensional algebra; see [4, Example 3]. This implies that the problem of finite-dimensional bases for $D^{k}$ is distinct from the equivalence problem.) The third section of this paper shows the extent to which classical results on the equivalence probelm can be utilized in the Lie algebra basis problem.

The motivation for this problem came from the study of control systems, on $\mathbf{R}^{n}$, of the form

$$
\dot{x}=\sum_{i=1}^{k} u_{i} X^{i}(x), \quad x(0)=p
$$

with the controls $u_{i}$ taking values in $\mathbf{R}^{1}$. This system depends only on the distribution $D^{k}(x)=\operatorname{span}\left\{X^{1}(x), \ldots, X^{k}(x)\right\}$ and not on the specific basis 
chosen for $D^{k}$. Indeed, the use of control "feedback" $u_{i}(x)=\sum_{j=1}^{k} a_{i j}(x) v_{j}$, $i=1, \ldots, k$, with $A=\left(a_{i j}\right)$ a nonsingular, matrix-valued, function leads to the equivalent system $\dot{x}=\sum_{j=1}^{k} v_{j} Y^{j}(x)$ where $Y^{j}(x)=\sum_{i=1}^{k} a_{i j}(x) X^{i}(x)$, i.e. this is merely a basis change for $D^{k}$. If $D^{k}$ admits a nilpotent (or solvable) basis the analysis of (1) is greatly simplified. Indeed, see [5], [6, §3], in this case the solutions for specific control choices, can be written as the compositions of flows corresponding to the vector fields in a properly chosen, ordered basis, for the algebra $L\left(X^{1}, \ldots, X^{k}\right)$.

Systems of the form $(0.1)$ arise naturally in the control of manipulator linkages. A less standard origin is in singular differential equations, on $\mathbf{R}^{n}$, of the form

$$
B(x) \dot{x}=f(x), \quad x(0)=p
$$

where $B$ is an $n \times n$ matrix valued function with rank $B(x)=n-k$ for $x$ in a $n b d$ of $p$. If $D^{k}(x)$ denotes the null space of $B(x)$, the general solution of $(0.2)$ is the sum of a specific solution of the nonhomogeneous problem with any solution of $\dot{x} \in D^{k}(x)$, this latter equation being of the form (0.1).

A final application is to the area of partial differential operators of the form $T w=\sum_{i=1}^{k}\left(X^{i}\right)^{2} w$ where $w: \mathbf{R}^{n} \rightarrow \mathbf{R}^{1}$. The operator $T$ is hypoelliptic (at $p$ ) if $\operatorname{dim} L\left(X^{1}, \ldots, X^{k}\right)(p)=n$. The fundamental singularity of the parametric for the problem $T w=f$ depends on $D^{k}(x)=\operatorname{span}\left\{X^{1}(x), \ldots, X^{k}(x)\right\}$ and not on the specific basis chosen. The study of such a parametric involves an analysis of the shape of "geodesic balls" for a system such as $(0.1)$ with control bounds $\left|u_{i}\right| \leq 1$. In control language, this is the shape of the attainable set at time $t=1$. A detailed analysis can be found in [7].

Thourghout this paper we work in the real analytic category. For $X$ a vector field on $M$ the notation $(\exp s X)(p)$ will denote the solution, at time $s$, of $\dot{x}=X(x), x(0)=p$.

\section{$\S 1$. The Theorem of Nagano-SussmanN}

The papers [2,3] study the equivalence question for Lie algebras of vector fields. Specifically, given transitive Lie algebras $L, L^{\prime}$ on manifolds $M, M^{\prime}$, respectively, and a Lie algebra isomorphism $\bar{l}: L \rightarrow L^{\prime}$, when is $\bar{l}$ induced by a diffeomorphism? The local version of the main theorm in [3] may be stated as follows.

Theorem (Sussman [3]). Let $L, L^{\prime}$ be Lie algebras of real analytic vector fields on the real analytic, $n$-dimensional manifolds $M, M^{\prime}$, respectively, and $p \in$ $M, q \in M^{\prime}$ such that $\operatorname{dim} L(p)=\operatorname{dim} L^{\prime}(q)=n$. If there is a Lie algebra isomorphism $l: L_{p} \rightarrow L_{q}^{\prime}$ which extends to an isomorphism $\bar{l}: L \rightarrow L^{\prime}$, then there exist neighborhoods, $U(p)$ and $U^{\prime}(q)$ and an analytic diffeomorphism $\phi: U(p) \rightarrow U^{\prime}(q)$ with $\phi(p)=q$ and $\phi_{*}(x) X(x)=(\bar{l} X)(\phi(x))$ for all $X \in L$, $x \in U(p)$. 
By choosing $L=L^{\prime}$ and $M=M^{\prime}$, this theorem has immediate applications to the equivalence and self-equivalence problem for a distribution $D^{k}$. For later reference, we state this as

Corollary 1.1. Let $D^{k}$ be a real analytic, $k$-distribution having local basis $\left\{X^{1}, \ldots, X^{k}\right\}$ defined in a nbd $U(p)$ with $L=L\left(X^{1}, \ldots, X^{k}\right)$ such that $\operatorname{dim} L(p)=n$. If $q$, sufficiently near $p$, is such that $L_{p}$ is isomorphic to $L_{q}$ and the isomorphism $l$ extends to an automorphism $\bar{l}$ of $L$, then $D^{k}(p)$ is equivalent to $\left(\bar{l} D^{k}\right)(q)=\operatorname{span}\left\{\left(\bar{l} X^{1}\right)(q), \ldots,\left(\bar{l} X^{k}\right)(q)\right\}$. If, furthermore,

$$
\left(\bar{l} X^{i}\right)(y) \in D^{k}(y), \quad i=1, \ldots, k ; \quad y \in U(q)
$$

then $D^{k}(p)$ is self-equivalent to $D^{k}(q)$.

Remark. 1. If $L\left(X^{1}, \ldots, X^{k}\right)$ is nilpotent, it was shown in [1] that every $n b d$ of $p$ contains a point $q \neq p$ such that $L_{p}=L_{q}$. In this case one may choose $\bar{l}=\mathrm{id}$ in Corollary 1.1 which then yields $D^{k}(p)$ self-equivalent to $D^{k}(q)$.

\section{$\S 2$. Distributions With SOlvable bases}

In this section we will prove, via a series of short propositions, the following. Theorem 2. Let $L=L\left(X^{1}, \ldots, X^{k}\right)$ be a solvable Lie algebra of vector fields on $M=M^{n}$ with $\operatorname{dim} L(p)=n$. Then in every $n b d$ of $p$ there is a point $q \neq p$ such that the isotropy subalgebras $L_{p}, L_{q}$ are isomorphic. Furthermore, if $l: L_{p} \rightarrow L_{q}$ is the Lie algebra isomorphism, $l$ extends to a Lie algebra automorphism $\bar{l}$ of $L$.

For $L=L\left(X^{1}, \ldots, X^{k}\right)$ solvable, and say of dimension $m$ over the reals, one can find (see [8, Corollary 3.7.5]) an ordered basis $\left\{Y^{1}, \ldots, Y^{m}\right\}$ such that for each $1 \leq s \leq m, L\left(Y^{1}, \ldots, Y^{s}\right)$ is an ideal, denoted $G_{s}$, in $L$. Specifically, there exist real-valued functions $\lambda_{j}: L \rightarrow \mathbf{R}$ such that

$$
\left[X, Y^{s}\right]=\lambda_{s}(X) Y^{s}+\sum_{j=1}^{s-1} \lambda_{j}(X) Y^{j}, \quad s=1, \ldots, m, \quad X \in L .
$$

Choose such a basis and let $r$ denote the smallest integer such that $Y^{r}(p) \neq 0$. Such an $r$ exists since $\operatorname{dim} L(p)=n$. We then have

Proposition 1.

$$
\begin{gathered}
{\left[X, Y^{r}\right]=\lambda_{r}(X) Y^{r}+\sum_{j=1}^{r-1} \lambda_{j}(X) Y^{j}, \quad X \in L .} \\
{\left[X, Y^{r}\right](p)=\lambda_{r}(X) Y^{r}(p), \quad \text { i.e. } \sum_{j=1}^{r-1} \lambda_{j}(X) Y^{j} \in G_{r-1} \subset L_{p} .} \\
\lambda_{r}([X, Z])=0 \quad \text { for all } X, Z \in L .
\end{gathered}
$$


Proof. Statements (2.1) and (2.2) are immediate from the choice of the ordered basis and integer $r$. For (2.3) we use the Jacobi identity, i.e.

$$
\begin{aligned}
{\left[[X, Z], Y^{r}\right]=} & {\left[X,\left[Z, Y^{r}\right]\right]-\left[Z,\left[X, Y^{r}\right]\right] } \\
= & {\left[X, \sum_{j=1}^{r} \lambda_{j}(Z) Y^{j}\right]-\left[Z, \sum_{j=1}^{r} \lambda_{j}(X) Y^{j}\right] } \\
= & \sum_{j=1}^{r} \lambda_{j}(Z) \sum_{i=1}^{j} \lambda_{i}(X) Y^{i} \\
& -\sum_{j=1}^{r} \lambda_{j}(X) \sum_{i=1}^{j} \lambda_{i}(Z) Y^{i} \\
= & \sum_{i=1}^{r}\left(\sum_{j=i}^{r} \lambda_{i}(X) \lambda_{j}(Z)-\lambda_{j}(X) \lambda_{i}(Z)\right) Y^{i} .
\end{aligned}
$$

From this, one sees that the coefficient of $Y^{r}$, which is $\lambda_{r}([X, Z])$, is zero.

Proposition 2. $X \in L_{p}$ if and only if

$$
X\left(\left(\exp s Y^{r}\right)(p)\right)=s \lambda_{r}(X) Y^{r}((\exp s Y)(p)), \quad s \text { real . }
$$

(In words, $X \in L_{p}$ if and only if along the trajectory of $Y^{r}$ thru $p, X$ is a specific scalar multiple of $Y^{r}$.)

Proof. (a) If (2.4) holds, then for $s=0$ we see $X \in L_{p}$.

(b) Suppose $X \in L_{p}$, i.e. $X(p)=0$. Then

$$
\begin{aligned}
\left(\exp -s Y^{r}\right)_{*} X\left(\left(\exp s Y^{r}\right)(p)\right) & =\sum_{\nu=0}^{\infty}\left((-s)^{\nu} / \nu !\right)\left(a d^{\nu} Y^{r}, X\right)(p) \\
& =X(p)+s\left[X, Y^{r}\right](p)=s \lambda_{r}(X) Y^{r}(p),
\end{aligned}
$$

where use was made of $(2.2)$ in the last equality. But $\left(\exp -s Y^{r}\right)_{*}$ is a vector space isomorphism and $\left(\exp -s Y^{r}\right)_{*} Y^{r}\left(\left(\exp s Y^{r}\right)(p)\right)=Y^{r}(p)$ hence it follows from (i) that $X\left(\left(\exp s Y^{r}\right)(p)\right)=s \lambda_{r}(X) Y^{r}\left(\left(\exp s Y^{r}\right)(p)\right)$.

Next, let $|s|>0$ be small and $q=\left(\exp s Y^{r}\right)(p)$. Define a map

$$
l: L_{p} \rightarrow L, \quad l(X)=X-s \lambda_{r}(X) Y .
$$

Proposition 3. The map $l$ is linear, $l: L_{p} \rightarrow L_{q}$ and is a Lie algebra isomorphism.

Proof. The linearity of $l$ follows immediately from its definition. Also, for $X \in L_{p}, l(X)(q)=X(q)-s \lambda_{r}(X) Y^{r}(q)$ which is zero by (2.4). Thus $l$ : $L_{p} \rightarrow L_{q}$.

Next, we show $l$ is one-one. Inded, for $X, Z \in L_{p}$ if $l(X)=l(Z)$, then $l(X)(p)=l(Z)(p) \Rightarrow \lambda_{r}(X)=\lambda_{r}(Z) \Rightarrow X=Z$. 
Finally, to see that $l$ is a Lie algebra isomorphism we show it preserves Lie products, i.e. that $l([X, Z])=[l(X), l(Z)]$. To save repeating this argument for the extension $\bar{l}$ of $l$, one may note that no use will be made of the fact that here $X, Z \in L_{p}$. First, $l([X, Z])=[X, Z]$ since $\lambda_{r}([X, Z])=0$ by $(2.3)$. Next, we compute

$$
\begin{aligned}
{[l(X), l(Z)] } & =\left[X-s \lambda_{r}(X) Y^{r}, Z-s \lambda_{r}(Z) Y^{r}\right] \\
& =[X, Z]-s \lambda_{r}(Z)\left[X, Y^{r}\right]+s \lambda_{r}(X)\left[Z, Y^{r}\right] \\
& =[X, Z]-s \lambda_{r}(X) \lambda_{r}(Z) Y^{r}+s \lambda_{r}(X) \lambda_{r}(Z) Y^{r}=[X, Z],
\end{aligned}
$$

as required.

We now extend $l$ to a mapping $\bar{l}: L \rightarrow L$ in the obvious way, i.e. define

$$
\bar{l}: L \rightarrow L, \quad \bar{l}(X)=X-s \lambda_{r}(X) Y^{r} .
$$

Proposition 4. The mapping $\bar{l}$ is an extension of $l$ and a Lie algebra automorphism of $L$.

Proof. From the definition, $\bar{l}$ agrees with $l$ in $L_{p}$. To see $\bar{l}$ is one-one, let $X, Z \in L$. Then $\bar{l}(X)=\bar{l}(Z) \Rightarrow X-s \lambda_{r}(X) Y^{r}=Z-s \lambda_{r}(Z) Y^{r} \Rightarrow(X-Z)=$ $s\left(\lambda_{r}(X)-\lambda_{r}(Z)\right) Y^{r} \Rightarrow(X(q)-Z(q))=s\left(\lambda_{r}(X)-\lambda_{r}(Z)\right) Y^{r}(q) \Rightarrow(X-Z) \in L_{p}$ (by Proposition 2) $\Rightarrow X(p)=Z(p) \Rightarrow \lambda_{r}(X)=\lambda_{r}(Z) \Rightarrow X=Z$.

The proof that $\bar{l}$ preserves Lie products is, as previously mentioned, the same as that given for $l$ in Proposition 3.

Propositions 1-4 complete the proof of Theorem 2. Indeed, $q$ can be chosen as any point on the (nonconstant) trajectory of $Y^{r}$ through $p$.

An immediate consequence of Corollary 1.1 and Theorem 2 is that if $D^{k}$ is a $k$-distribution on $M=M^{n}$ with local basis $\left\{X^{1}, \ldots, X^{k}\right\}$ such that $L=$ $L\left(X^{1}, \ldots, X^{k}\right)$ is solvable and $\operatorname{dim} L(p)=n$, then every $n b d$ of $p$ contains a point $q \neq p$ such that $D^{k}(p)$ is equivalent to $\bar{l} D^{k}(q)$ where $\bar{l} D^{k}(x)=$ $\operatorname{span}\left\{\left(\bar{l} X^{1}\right)(x), \ldots,\left(\bar{l} X^{k}(x)\right\}\right.$. Furthermore, we may note from the definition, (2.6), of $\bar{l}$ that if $Y^{r} \in D^{k}$, then $X^{i} \in D^{k} \Rightarrow \bar{l} X^{i} \in D^{k}$ and hence $\bar{l} D^{k}=D^{k}$ in which case we can conclude $D^{k}(p)$ is self-equivalent to $D^{k}(q)$. In general one cannot expect $Y^{r} \in D^{k}$, however, as we next show, for $q$ sufficiently near $p$, $\bar{l}$ is near the identity hence $D^{k}(q)$ and $\bar{l} D^{k}(q)$ have certain relevant invariants in common, specifically the dimensions of the derived distributions.

Theorem 3. Let $D^{k}$ be a $k$-distribution on $M^{n}$ with local basis $\left\{X^{1}, \ldots, X^{k}\right\}$ such that $L=L\left(X^{1}, \ldots, X^{k}\right)$ is solvable and $\operatorname{dim} L(p)=n$. Then every $n b d$ of $p$ contains a point $q \neq p$ such that $\operatorname{dim} D^{k, j}(p)=\operatorname{dim} D^{k, j}(q)$ for $j=$ $1,2, \ldots$.

Proof. Let $\bar{l}$ be the Lie algebra automorphism as given by (2.6) and $q \neq p$ a point such that $D^{k}(p),\left(\bar{l} D^{k}\right)(q)$ are equivalent. 
By Theorem 1, $\exists$ a local diffeomorphism $\phi$ defined on a $n b d$. $U(p)$ such that $\phi(p)=q$ and $\phi_{*}(x) D^{k}(x)=\bar{l} D^{k}(\phi(x)), x \in U(p)$. Since $\phi_{*}(x)\left[X^{i}, X^{j}\right]$ $\cdot(x)=\left[\bar{l} X^{i}, \bar{l} X^{j}\right](\phi(x))$ it clearly follows that $\operatorname{dim} D^{k, j}(p)=\operatorname{dim} \bar{l} D^{k, j}(q)$, $j=1, \ldots$, i.e. these dimensions are diffeomorphism invariants. What remains to be shown is that $\operatorname{dim} \bar{l} D^{k, j}(q)=\operatorname{dim} D^{k, j}(q)$ for $q$ sufficiently near $p$.

Now $\left(\bar{l} X^{i}\right)(q)=X^{i}(q)-s \lambda_{r}\left(X^{i}\right) Y^{r}(q)$ and $\left(\bar{l}\left[X^{i}, X^{j}\right]\right)(q)=\left[X^{i}, X^{j}\right](q)$, which follows since $\lambda_{r}\left(\left[X_{i}, X^{j}\right]\right)=0$ by (2.2). A well-known result of Dynkin (see [8; Ex. 44, p. 146]) shows when computing a generating set for $D^{k, j}$ it suffices to consider products of $s$-tuples of $X^{1}, \ldots, X^{k}$ of the special form $\left[X^{i_{1}},\left[X^{i_{2}},\left[\cdots\left[X^{i_{s-1}}, X^{i_{s}}\right] \cdots\right]\right]\right]$. Thus $D^{k, j}(q)=\operatorname{span}\left\{X^{1}(q), \ldots, X^{k}(q)\right.$, $\left.\left[X^{i_{1}},\left[\cdots\left[X^{i_{s-1}}, X^{i_{s}}\right] \cdots\right]\right](q) ; s \leq j\right\}$ while the $j$ th derived distribution of $\bar{l} D^{k}$, denoted $\left(\bar{l} D^{*}\right)^{j}$, satisfies

$$
\begin{aligned}
& \left(\bar{l} D^{k}\right)^{j}(q)=\operatorname{span}\left\{X^{1}(q)+s \lambda_{r}\left(X^{1}\right) Y^{r}(q), \ldots,\right. \\
& \left.\qquad X^{k}(q)+s \lambda_{r}\left(X^{k}\right) Y^{r}(q),\left[X^{i_{1}}\left[\cdots\left[X^{i_{s-1}}, X^{i_{s}}\right] \cdots\right]\right](q) ; s \leq j\right\} .
\end{aligned}
$$

From this, one sees for $|s|>0$ but sufficiently small (i.e. for $q=\left(\exp s Y^{r}\right)$ - (p) sufficiently near $p) \operatorname{dim} D^{k, j}(q)=\operatorname{dim} \bar{l} D^{k, j}(q)=\operatorname{dim} D^{k, j}(p)$, $j=1, \ldots$.

Corollary 1. If $D^{k}(x)=D^{k, 0}(x)=\operatorname{span}\left\{X^{1}(x), \ldots, X^{k}(x)\right\}$ is a local $k$ distribution on $M^{n}$ with $\operatorname{dim} L\left(X^{1}, \ldots, X^{k}\right)(p)=n$ and such that $\operatorname{dim} D^{k, 1}(p)$ $=k$ while $\operatorname{dim} D^{k, 1}(x)>k$ if $x \neq p$, then $D^{k}$ does not admit a solvable basis in any nbd of $p$.

In [1, Example 1.1], for every $2 \leq k \leq n-1$ such a $D^{k}$ was constructed hence one has

Corollary 2. For every $2 \leq k \leq n-1$ there exists a $k$-distribution $D^{k}$, defined on $\mathbf{R}^{n}$ which does not admit a solvable basis in any nbd of zero.

For completeness, we illustrate this via the construction of a $D^{2}$ on $\mathbf{R}^{3}$ which admits no solvable basis in any $n b d$ of zero.

Example 1. Choose $X^{1}(x)=\partial / \partial x_{1}, X^{2}(x)=\partial / \partial x_{2}-x_{1}\left(\left(x_{1}^{2} / 3\right)+x_{2}^{2}+\right.$ $\left.x_{3}^{2}\right) \partial / \partial x_{3}$ and $D^{2}(x)=\operatorname{span}\left\{X^{1}(x), X^{2}(x)\right\}$. Then $\left[X^{1}, X^{-2}\right](x)=\left(x_{1}^{2}+\right.$ $\left.x_{2}^{2}+x_{3}^{2}\right) \partial / \partial x_{3},\left(a d^{2}\left[X^{1}, X^{2}\right], X^{1}\right)(0)=2 \partial / \partial x_{3}$ hence $\operatorname{dim} L\left(X^{1}, X^{2}\right)(0)=3$ while

$$
\operatorname{dim} D^{2,1}(x)=\left\{\begin{array}{l}
2 \text { if } x=0 \\
3 \text { if } x \neq 0
\end{array}\right.
$$

By Corollary $1, D^{2}$ does not admit a solvable basis in any $n b d$ of zero.

\section{§3. The ROLE OF THE EQUiVAlENCE PROBLEM IN THE BASIS PROBLEM}

The equivalence problem for (locally defined) $k$-distributions on $M^{n}$ may be viewed as their classification up to local diffeomorphisms. This problem was studied in the dual setting by Cartan [9], Goursat [10], Darboux, Von 
Webber, etc. Given $D^{k}(x)=\operatorname{span}\left\{X^{1}(x), \ldots, X^{k}(x)\right\}$ defined in a $n b d U$ of $p \in M^{n}$ let $\omega_{1}, \ldots, \omega_{n-k}$ be linearly independent one-forms on $M^{n}$ such that $\left\langle\omega_{i}(x), X^{j}(x)\right\rangle=0, i=1, \ldots, n-k, j=1, \ldots, k$, for $x \in U$. Then the module $I_{n-k}$ generated by $\omega_{1}, \ldots, \omega_{n-k}$ over the ring of smooth, real valued, functions on $M^{n}$ is called the dual pfaffian system to $D^{k}$. For example, if $k=n-1, I_{1}$ is generated by a single one form $\omega$. The rank of $\omega$ at $x$ is one-half the rank of the $(n-1) \times(n-1)$ skew symmetric matrix $S(x)=$ $\left(\left\langle\omega(x),\left[X^{i}, X^{j}\right](x)\right\rangle\right)$. If $\omega$ has constant rank in a $n b d$ of $p$, Dabroux's theorem gives a canonical form for $\omega$ relative to a specific choice of local coordinates. This, in turn, allows one to make an obvious choice of a dual vector field basis for $D^{n-1}$ such that the elements of this basis generate a nilpotent Lie algebra. (See [1].) Thus although the Lie algebra structure of a vector field basis is independent of the local coordinates used to represent the basis elements, and although the equivalence classification does not take into account any specific bases, the classical results can give positive information concerning the existence of bases generating nice Lie algebras.

The classification of low dimensional pfaffian systems in the classical work of Cartan, Darboux, etc. was based on invariants, e.g. the rank $\omega(x)$ in the Darboux theorem, which were required to be locally constant. These invariants can be interpreted in the dual, or distribution, setting; our next task.

For any $k$-distribution $D^{k}$ on $M^{n}$ define its Cauchy subdistribution $C\left(D^{k}\right)=\left\{X \in D^{k}:\left[X, D^{k}\right] \subset D^{k}\right\}$. The Jacobi identity readily shows $C\left(D^{k}\right)$ is involutive. Again, the $D^{k, j}$ denote derived distributions. The invariants used by Cartan, Dabroux, etc. to classify pfaffian systems can be defined in terms of $\operatorname{dim} D^{k, j}(x), \operatorname{dim} C\left(D^{k, j}\right)(x)$ and hence to use the classical results, these dimensions should be locally constant. With this in mind we define $D^{k}$ on $M^{n}$ to be regular at $p \in M^{n}$ if for each $j \geq 0, \operatorname{dim} D^{k, j}(x), \operatorname{dim} C\left(D^{k, j}\right)(x)$ are constant in a $n b d$ of $p$. One should remark that this is a slightly stronger notion of regular than that used by Hermann in [13]. Indeed, in [13], only $\operatorname{dim} D^{k, j}(x)$ locally constant was required while the invariants $C\left(D^{k, j}\right)(x)$ were given an interesting characterization via integrability tensors.

For illustration, if $D^{n-1}$ on $\mathbf{R}^{n}$ is regular at zero in the above sense and $\omega$ is a nonzero, dual, one form then (see [11, Theorem 1]) we have $2(\operatorname{rank} \omega(x))=$ $(n-1)-\operatorname{dim} C\left(D^{n-1}\right)(x)$. Thus $D^{n-1}$ regular implies rank $\omega(x)$ is locally constant and the Darboux theorem applies. It is worth noting that the dimensions of Cauchy subdistributions are computable via linear algebra, see [11].

Example 2. $\left(\operatorname{dim} D^{k, j}(x)\right.$ locally constant but $\operatorname{dim} C\left(D^{k, j}\right)(x)$ not locally constant.)

Let $h(x)=-x_{3} x_{1}^{2}-x_{3} x_{2}^{2}-\left(x_{3}^{2} / 2\right)-x_{3} x_{4}^{2}-x_{3} x_{5}^{2}$. We consider $D^{4}(x)=$ $\operatorname{span}\left\{X^{1}(x), \ldots, X^{4}(x)\right\}$ on $\mathbf{R}^{5}$ with $X^{1}=\partial / \partial x_{1}, X^{2}=\partial / \partial x_{2}-x_{1} \partial / \partial x_{5}$, $X^{3}=\partial / \partial x_{3}, X^{4}=\partial / \partial x_{4}+h(x) \partial / \partial x_{5}$. Since $\left[X^{1}, X^{2}\right]=\partial / \partial x_{5}, \operatorname{dim} D^{4, j}(x)$ $=5$ for $j \geq 1$ and $x$ in a $n b d$ of $0 \in \mathbf{R}^{5}$. A dual, nonzero, one form is 
$\omega(x)=x_{1} d x_{2}-h(x) d x_{4}+d x_{5}$ and a calculation shows

$$
\operatorname{rank} S(x)=\operatorname{rank}\left(\left\langle\omega(x),\left[X^{i}, X^{j}\right](x)\right\rangle\right)_{1 \leq i, j \leq 4}=\left\{\begin{array}{l}
2 \text { if } x=0 \\
4 \text { if } x \neq 0
\end{array}\right.
$$

Thus

$$
\operatorname{dim} C\left(D^{4}\right)(x)=\left\{\begin{array}{l}
2 \text { if } x=0 \\
0 \text { if } x \neq 0
\end{array}\right.
$$

Here, $D^{4}$ is not regular.

A first example of the use of the classical equivalence results in the nilpotent basis problem can be stated as

Proposition 5. ([1]). Every $D^{n-1}$ on $M^{n}$ which is regular at $p$ admits a local, nilpotent basis which generates an algebra of dimension $n$.

As a next example, we have

Proposition 6. Every $D^{k}$ on $M^{4}, 1 \leq k \leq 4$, which is regular at $p$ admits a local, nilpotent, basis.

Proof. For $k=1$ or $4, D^{k}$ is involutive hence has an Abelian basis; for $k=3$ we may use Proposition 5 hence only the case $k=2$ remains.

From $[10, \S 75]$, the dual pfaffian system $I_{2}$ to $D^{2}$ may be taken to have generators $\omega_{1}, \omega_{2}$ which in preferred local coordinates take one of the following forms:

(A) $\omega_{1}=-y_{3} d y_{1}+d y_{2}, \omega_{2}=-y_{4} d y_{1}+d y_{3}$,

(B) $\omega_{1}=d y_{1}, \omega_{2}=-y_{4} d y_{2}+d y_{3}$,

(C) $\omega_{1}=d y_{1}, \omega_{2}=d y_{2}$.

For each case we choose a specific basis for the dual distribution $D^{2}$, i.e. respectively:

$\left(\mathrm{A}^{\prime}\right) D_{1}^{2}(y)=\operatorname{span}\left\{X^{1}(y)=\partial / \partial y_{4}, X^{2}(y)=\partial / \partial y_{1}+y_{3} \partial / \partial y_{2}+y_{4} \partial / \partial y_{3}\right\}$,

(B') $D_{2}^{2}(y)=\operatorname{span}\left\{X^{1}(y)=\partial / \partial y_{4}, X^{2}(y)=\partial / \partial y_{2}+y_{4} \partial / \partial y_{3}\right\}$,

(C') $D_{3}^{2}(y)=\operatorname{span}\left\{X^{1}(y)=\partial / \partial y_{4}, X^{2}(y)=\partial / \partial y_{3}\right\}$.

One may note that in case $\left(\mathrm{C}^{\prime}\right)$ we have $D_{3}^{2}$ involutive hence the Frobenius theorem gives the Abelian basis. In case $\left(\mathbf{B}^{\prime}\right),\left[X^{1}, X^{2}\right]=\partial / \partial x_{3}$ and all other brackets vanish. Here $\operatorname{dim} D_{2}^{2,1}(x)=3$ and $D_{2}^{2,1}$ is involutive. Thus $D_{2}^{2}$ can be considered as a two distribution on the three-dimensional leaves of $D_{2}^{2,1}$ and the Darboux theorem can be used. Finally, in case $\left(\mathrm{A}^{\prime}\right), \operatorname{dim} D_{1}^{2,1}(x)=3$, $\operatorname{dim} D_{1}^{2,2}(x)=4$ which is sometimes called the Goursat-Von Weber case (see [12, Theorem 4.4]). In every case, the bases chosen generate a nilpotent Lie algebra.

Notice that the counterexamples to the existence of a nilpotent basis in [1] and to a solvable basis in $\S 2$, here, depended on $D^{k}$ being not regular. 
The case of $D^{2}$ on $M^{5}$. The equivalence problem for two- and three-dimensional pfaffian systems in five variables was extensively studied by Cartan [9]; the results are also given by Goursat [10]. Dual to a three-dimensional pfaffian system on $M^{5}$ is a two-dimensional distribution which we will next analyze in detail. Similar results could be obtained for $D^{3}$ on $M^{5}$. We will use invariants of the distribution $D^{k}$; Cartan and Goursat use invariants of the dual pfaffian system $I_{n-k}$, mainly the class of $I_{n-k}$ which is $\operatorname{dim} C\left(D^{k}\right)$. This invariant may also be defined via short exact sequences associated with $I_{n-k}$, see [14].

Let $D^{2}$ be a two distribution defined in a $n b d$ of $0 \in \mathbf{R}^{5}$; be regular at zero and have dual pfaffian system denoted $I_{3}$, with class $I_{3}=5$ (i.e., $\operatorname{dim} C\left(D^{2}\right)(x)=0$ in a $n b d$ of zero which is the generic case). Then if $D^{2}(x)=\operatorname{span}\left\{X^{1}(x), X^{2}(x)\right\}$ we know $\left[X^{1}, X^{2}\right] \notin D^{2}$ i.e., $\operatorname{dim} D^{2,1}(x)=3$. Dual to $D^{2,1}$ is the first derived pfaffian system which will necessarily be generated by two independent one forms. Following the notation of [10], we denote this as $I_{2}^{\prime}$. The classification (canonical forms) of $I_{3}$, and hence $D^{2}$, depend on the class of $I_{2}^{\prime}$, i.e. $\operatorname{dim} C\left(D^{2,1}\right)$. The possibilities are (see also [10, p. 317]):

(E) $\operatorname{dim} C\left(D^{2,1}\right)=0$, i.e. $\left(a d^{2} X^{1}, X^{2}\right) \notin D^{2,1},\left(a d^{2} X^{2}, X^{1}\right) \notin D^{2,1}$.

(F) $\operatorname{dim} C\left(D^{2,1}\right)=1$; one of $\left(a d^{2} X^{1}, X^{2}\right),\left(a d^{2} X^{2}, X^{1}\right)$ belongs to $D^{2,1}$.

(G) $\operatorname{dim} C\left(D^{2,1}\right)=3 ; X^{1}, X^{2},\left[X^{1}, X^{2}\right]$ are involutive.

Case $(G)$. (See [10, p. 318].) Here one can choose a basis for $I_{3}$ of the form $\omega_{1}=d y_{1}, \omega_{2}=d y_{2}, \omega_{3}=-y_{5} d y_{3}+d y_{4}$ hence in properly chosen local coordinates $D^{2}$ has a basis $\left\{X^{1}(y)=\partial / \partial y_{5}, X^{2}(y)=\partial / \partial y_{3}+y_{5} \partial / \partial y_{4}\right\}$ and this basis generates a nilpotent Lie algebra.

Case (F). (See $\left[10\right.$, p. 318].) Here $I_{3}$ has either a basis $\omega_{1}=d y_{1}, \omega_{2}=$ $y_{4} d y_{2}-d y_{3}, \omega_{3}=y_{5} d y_{2}-d y_{4}$ and $D^{2}$ has $\left\{X^{1}=\partial / \partial y_{5}, X^{2}=\partial / \partial y_{2}+\right.$ $\left.y_{4} \partial / \partial y_{3}+y_{5} \partial / \partial y_{4}\right\}$ as a nilpotent basis or $I_{3}$ has basis $\omega_{1}=-y_{3} d y_{1}+d y_{2}$, $\omega_{2}=-y_{4} d y_{1}+d y_{2}, \omega_{3}=-y_{5} d y_{1}+d y_{4}$ and $D^{2}$ has $\left\{X^{1}=\partial / \partial y_{5}, X^{2}=\right.$ $\left.\partial / \partial y_{1}+y_{3} \partial / \partial y_{2}+y_{4} \partial / \partial y_{3}+y_{5} \partial / \partial y_{4}\right\}$ as a nilpotent basis.

Case $(E)$. (See $\left[10\right.$, p. 320].) In this case $I_{3}$ was called normal; it is the generic case and $I_{3}$ has a basis of the form $\omega_{1}=-y_{3} d y_{1}+d y_{2}, \omega_{2}=-f d y_{1}-$ $y_{5} d y_{3}+d y_{4}, \omega_{3}=\left(\partial f / \partial y_{5}\right) d y_{1}+d y_{3}$ where $f=f\left(y_{1}, \ldots, y_{5}\right)$ may be arbitrary except for $\partial^{2} f(0) / \partial y_{5}^{2} \neq 0$. Here $D^{2}$ has, as a canonical basis:

$$
\left\{X^{1}=\frac{\partial}{\partial y_{5}}, X^{2}=\frac{\partial}{\partial y_{1}}+y_{3} \frac{\partial}{\partial y_{2}}-\left(\frac{\partial f}{\partial y_{5}}\right) \frac{\partial}{\partial y_{3}}+\left(f-y_{5} \frac{\partial f}{\partial y_{5}}\right) \frac{\partial}{\partial y_{4}}\right\} \text {. }
$$

It is easy to see that one can choose functions $f$, with $\partial^{2} f(0) / \partial y_{5}^{2} \neq 0$, in (3.1) such that the Lie algebra generated by $X^{1}, X^{2}$ is infinite dimensional. This does not mean, for such an $f$, that a change of basis could not yield a new basis generating a finite Lie algebra, i.e. the Lie algebra basis problem becomes distinct from the equivalence problem. 


\section{REFERENCES}

1. H. Hermes, A. Lundell and D. Sullivan, Nilpotent bases for distributions and control systems, J. Differential Equations 55 (1984), 385-400.

2. T. Nagano, Linear differential systems with singularities and an application of transitive Lie algebras, J. Math. Soc. Japan 18 (1966), 398-404.

3. H. Sussmann, An extension of a theorem of Nagano on transitive Lie algebras, Proc. Amer. Math. Soc. 45 (1974), 349-356.

4. H. Hermes, Distributions having bases which generate finite dimensional Lie algebras, Systems Control Lett. 8 (1987), 375-380.

5. K. T. Chen, Decomposition of differential equations, Math. Ann. 146 (1962), 263-278.

6. H. Hermes, Local controllability and sufficient conditions in singular problems. II, SIAM J. Contraol Optim. 14 (1976), 1049-1062.

7. A. Nagle, E. M. Stein and S. Wainger, Balls and metrics defined by vector fields. I. Basic Properties, Acta Math. 155 (1985), 103-147.

8. V. S. Varadarajan, Lie groups, Lie algebras, and their representations, Prentice-Hall, Inc., Englewood Cliffs, N.J., 1974.

9. M. E. Cartan, Systèmes de Pfaff a Cinq Variables; Oeuvres Complètes, Partie II, GauthierVillars, Paris 1953.

10. E. Goursat, Lecons sur le Probleme de Pfaff, Hermann Pub., Paris, 1922.

11. H. Hermes, Involutive subdistributions and canonical forms for distributions and control systems, Theory and Applications of Nonlinear Control Systems, Byrnes, Lindquist, Eds., Elsevier Pub., North Holland, 1986.

12. R. Gardner, Differential geometric methods interfacing control theory, Differential Geometric Control Theory (Brockett-Millman-Sussman, editors), Birkhäuser, Boston, 1983, 117-180.

13. Robert Hermann, The theory of equivalence of pfaffian systems and input systems under feedback, Math. Systems Theory 15 (1982), 343-356.

14. R. Gardner, Invariants of pfaffian systems, Trans. Amer. Math. Soc. 126 (1967), 514-533.

Mathematics Department, Box 426, University of Colorado, Boulder, COLORADO 80309 